\title{
HIGH ELECTRON CYCLOTRON HARMONIC EMISSIONS FROM AURORA
}

\author{
J. LaBelle*
}

\begin{abstract}
The aurora is characterized by highly non-equilibrium electron distributions that excite a variety of plasma waves, some of which result in radio emissions that can be detected at ground-level. In the frequency range between $50 \mathrm{kHz}$ and $10 \mathrm{MHz}$, five distinct types have been identified: auroral hiss, auroral kilometric radiation (AKR), auroral roar (electron cyclotron harmonic emissions), auroral medium frequency burst (MFB), and recently discovered auroral emissions just above the electron cyclotron frequency. The last few years have seen significant advances in all of these emissions. Regarding auroral roar emissions, for example, the exciting recent development has been the discovery of emissions at relatively high harmonics of the electron cyclotron frequency. In contrast to the lower harmonic emissions which are always left-polarized, the higher harmonic emissions are sometimes left, sometimes right polarized, with polarization and frequency distribution related to whether the emissions occur under daylight or darkness conditions. Studies of the fine structure of multiple harmonics reveal the emissions to sometimes be harmonically related, sometimes not, again with a relation to daylight or darkness conditions. These studies suggest that two different mechanisms act to generate two distinct types of cyclotron harmonic radiation, one involving linear excitation and mode conversion of upper hybrid waves, and the other involving nonlinear wave-wave interactions. These results are interesting because they identify a naturally occurring nonlinear process in the near-Earth environment.
\end{abstract}

\footnotetext{
* Department of Physics and Astronomy, Dartmouth College, Hanover, NH, USA
} 
